\title{
Fully frustrated simple cubic lattices and the overblocking effect
}

\author{
B. Derrida \\ Institut Laue-Langevin, 38042 Grenoble, France
}

Y. Pomeau

Service de Physique Théorique, C.E.N. Saclay, 91190 Gif sur Yvette, France

G. Toulouse and J. Vannimenus

Laboratoire de Physique de l'Ecole Normale Supérieure, 24, rue Lhomond, 75231 Paris Cedex 05, France

(Reçu le 6 novembre 1978, révisé le 5 février 1979, accepté le 13 février 1979)

\begin{abstract}
Résumé. - Après l'étude des réseaux cubiques à faces centrées complètement frustrés par S. Alexander et P. Pincus, on étudie les réseaux cubiques simples, afin de déterminer quelles sont les propriétés universelles de tels modèles d'Ising. On montre que l'effet de surblocage, apparition obligatoire de plaquettes surfrustrées due à des contraintes géométriques, prend place, pour les réseaux cubiques simples aussi, au-delà de la dimension quatre. Il est montré que la moyenne' du champ interne dans l'état de base se comporte similairement dans les deux familles de réseaux. D'autres aspects sont trouvés différents : pas de périodicité en dimension (au lieu d'une périodicité deux), dégénérescence de l'état de base. Une procédure d'échelle permet de construire explicitement des états de base jusqu'en dimension 7 pour les réseaux cubiques simples et en toute dimension pour les réseaux cfc. Des bornes sont obtenues en dimension $\geqslant 8$ pour l'énergie de l'état de base des réseaux cubiques simples. Le réseau diamant est aussi discuté.
\end{abstract}

\begin{abstract}
Fully frustrated simple cubic (sc) lattices are investigated, following the study of face centred cubic lattices by S. Alexander and P. Pincus, in order to determine what are the universal properties of such Ising models. The overblocking effect, compulsory appearance of overfrustrated plaquettes due to geometric hindrances, is shown to take place for sc lattices also, above dimension four. The ground state average internal field is shown to behave similarly in both lattice families. Other features are found to differ : no periodicity in dimension (instead of periodicity two), ground state degeneracy. A scaling procedure allows explicit constructions to be made of ground states up to $d=7$ for sc lattices, and in any dimension for fcc lattices. Upper and lower bounds for the ground state energies of sc lattices are given for $d \geqslant 8$. The diamond lattice is also discussed.
\end{abstract}

Introduction. - In the theoretical models of spin glasses, two main ingredients are introduced : disorder and frustration [1]. Since the mutual presence of these two ingredients creates appreciable complexity, it is natural to analyse the effects of one without the other. Disorder without frustration allows an exact treatment of spin glass models to be made but the solution is too simple to be of real interest. Frustration without disorder is a more promising line of attack, which has been explored by J. Villain [2] (after earlier works concerned with antiferromagnetic structures), and where further progress has been made recently by S. Alexander and P. Pincus [3].

In these works, periodic lattices with nearest neighbour interactions are considered such that all ele- mentary plaquettes are frustrated (that is, fully frustrated models). J. Villain has considered some two and three dimensional lattices, for which he has presented a number of results and conjectures. By considering the family of face centred cubic fully frustrated lattices in arbitrary dimension, S. Alexander and P. Pincus have been able to unravel a striking phenomenon : whereas, for $d \leqslant 4$, the ground state of an Ising fully frustrated fcc model is such that each elementary plaquette (in these lattices, those plaquettes are triangles) contains at most one frustrated bond (minimum number allowed by the frustration), on the other hand, for $d>4$, any Ising ground state contains a finite density of triangles with three frustrated bonds. This means that there is a collec- 
tive effect which prevents at least a fraction of plaquettes from reaching their minimal energy states.

To this effect we shall give the name of overblocking effect because, besides the frustration which blocks a plaquette out of configurations without frustrated bond, this overblocking effect further blocks plaquettes out of their minimally frustrated states.

This remarkable fact, discovered by S. Alexander and P. Pincus, raises naturally two questions, which receive a partial answer below. The first question is whether the overblocking effect is lattice-dependent, or of sufficient universality to be of interest for a discussion of disordered systems. The second question is whether it is possible to obtain a simple geometric understanding of the overblocking effect.

By studying the family of simple cubic fully frustrated lattices in arbitrary dimension, with an approach best suited for these lattices and quite different from the Alexander-Pincus approach which was best suited for fcc lattices (see however Appendix II), we are able to show the generality of the overblocking effect and to provide a simple geometrical picture for it.

The results are probably best described in terms of the internal field defined on each lattice site (this field is the difference between the numbers of unfrustrated and frustrated bonds adjacent to one site). Whereas for low dimensions $(d \leqslant 4)$, the average internal field in the ground states is proportional to the coordination number (number of nearest neighbours), above dimension four, the overblocking effect takes place, a saturation of the internal field appears and its behaviour for large $d$ is proportional to the square root of the coordination number.

In order not to confuse the issue, the Ising case alone, which is important enough, will be discussed here.

1. Preliminary remarks on lattice structures. - In the square and diamond fully frustrated lattices studied by J. Villain, the symmetry of the underlying Bravais lattice is necessarily broken by the introduction of frustration. This led rather naturally S. Alexander and P. Pincus to consider fcc lattices, because in this case the antiferromagnet is fully frustrated and therefore the diagonalization of the interaction matrix and the generalization to arbitrary dimension are easy.

We want to show here that the case of simple cubic fully frustrated lattices turns out to have some simplicity of its own, despite the increase of the unit cell due to frustration, because one can actually reason entirely within the unit cell of the underlying Bravais lattice, which is the simplest possible $d$-dimensional cell that is a hypercube.

As we shall see, the case of the diamond lattice (in dimension 3 ) is a bit more difficult to solve, and this explains why the conjecture of Villain on the Ising ground state energy is indeed unverified.

When we consider lattices with plaquettes contain- ing an even number of bonds, our first task is to show that a fully frustrated lattice is actually realizable (if the number is odd, as in fcc lattices, the antiferromagnet provides an answer). Our second task is to obtain the Ising ground state energy for such a fully frustrated lattice.

These two tasks can be performed in one step if we look for the configuration of bonds with the minimal number of negative bonds which does fully frustrate the lattice. The Ising ground state energy will then be obtained for the state with all spins ferromagnetically aligned, and will be simply related to this minimal number of negative bonds (this is a direct consequence of the gauge transformation properties).

Now in the case of simple cubic lattices, it turns out that this search needs to be done only for the unit hypercube, a lattice configuration of bonds being simply obtained from the hypercube configuration of bonds by successive reflexions with respect to the various hyperplanes limiting the unit hypercube.

2. Detailed treatment. - We consider the Hamiltonian

$$
\mathscr{H}=-\sum_{i j} J_{i j} S_{i} S_{j}
$$

where $S_{i}$ is an Ising spin, on site $i$, taking \pm 1 values, and where only nearest neighbour interactions are considered. The energy associated with a bond can take two values : $+J$ (if the bond is frustrated) or $-J$ (if it is unfrustrated).

The lattice structure is, for our present purpose, supposed to be of the simple cubic type in arbitrary dimension $d$. The unit cell is therefore the unit hypercube in $d$ dimensions, which we shall call $\mathrm{H}_{d}$. For $d=2, \mathrm{H}_{2}$ is just a square; for $d=3, \mathrm{H}_{3}$ is just an ordinary cube. $\mathrm{H}_{d}$ is a regular $d$-dimensional polytope (polygon for dimension 2, polyhedron for dimension 3), and this fact is the reason for the simplicity of the treatment. As a matter of fact, the regularity of $\mathrm{H}_{d}$ means that all sites of the unit cell are equivalent and the various countings are hence simplified (note that fcc lattices do not have this sor $t$ of simplicity).

As announced in the previous section, we shall try and build a fully frustrated $\mathrm{H}_{d}$, with the minimal number of negative bonds. Let us first consider what a $\mathrm{H}_{d}$ is made of. $\mathrm{A} \mathrm{H}_{d}$ contains $n_{0}=2^{d}$ sites (or 0 cells), $n_{1}=2^{d-1} . d$ bonds (or 1-cells),

$$
n_{2}=2^{d-3} d(d-1)
$$

plaquettes (or 2-cells), and more generally $n_{p}=2^{d-p} C_{d}^{p}$ ( $p$-cells). Note that these $p$-cells are just $p$-dimensional hypercubes $\mathrm{H}_{p}$. We can check that the numbers $n_{p}$ verify the Euler-Poincare relation :

$n_{0}-n_{1}+n_{2}+\cdots+(-1)^{p} n_{p}+\cdots+(-1)^{d}=1$. 
Remark also that there is a notion of opposite : a site, a bond, a plaquette, ..., a $\mathrm{H}_{d-1}$ have an opposite within the hypercube $\mathrm{H}_{d}$.

Now it is readily observed that a fully frustrated $\mathrm{H}_{2}$ (square) can be made with one negative bond (Fig. 1), and that a fully frustrated $\mathrm{H}_{3}$ (cube) can be made with three negative bonds (Fig. 2). But, since the number of plaquettes rises faster with dimensionality $d$ than the number of bonds, it is not a priori obvious that it is possible to build a fully frustrated $\mathrm{H}_{d}$ for arbitrarily high $d$. However, this result can be proved by the following simple recurrence construction.

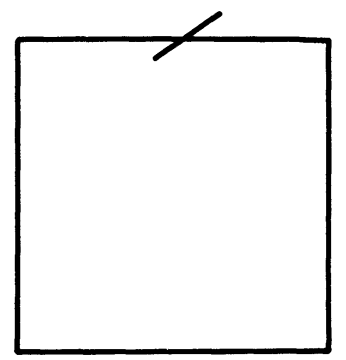

Fig. 1. - Fully frustrated $\mathrm{H}_{2}$ (square) with one negative bond. By convention, positive bonds are unbarred and negative bonds are barred.

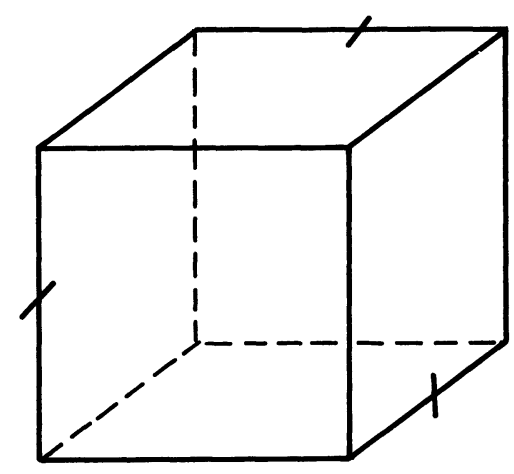

Fig. 2. - Fully frustrated $\mathrm{H}_{3}$ (cube) with three negative bonds.

Consider a $\mathrm{H}_{d}$ as built from two $\mathrm{H}_{d-1}$ by drawing bonds between their corresponding sites. Take for one of the two $\mathrm{H}_{d-1}$ a configuration of bonds which fully frustrate it (we suppose that this is possible), and for the other $\mathrm{H}_{d-1}$ the corresponding configuration but with all bond signs interchanged. Finally, take all bonds linking the two $\mathrm{H}_{d-1}$ as positive : with this configuration of bonds, obviously all plaquettes contain an odd number of negative bonds, and the full frustability of a $\mathrm{H}_{d}$ is thus proved for arbitrary dimension by recurrence.

This construction however does not yield low energy states, if we assume all spins to be ferromagnetically aligned. Therefore we must now look, among all bond configurations which fully frustrate $\mathrm{H}_{d}$, for those with a minimal number of negative bonds.

If we are able to find a fully frustrating bond configuration where each plaquette contains one and one only negative bond, then the Ising ground state energy (per spin in the lattice and per $J$ unit) will be

$$
E_{d}=-\frac{d}{2}
$$

(More generally, for a lattice with coordination number $z$ and $l$ bonds per plaquette, the formula will be

$$
E=-z \cdot \frac{l-2}{2 l}
$$

here $z=2 d$ and $l=4$.)

Now since the number of plaquettes is $2^{d-3} d(d-1)$, and since one bond belongs to $(d-1)$ plaquettes, we need at least $2^{d-3} d$ negative bonds in order to fully frustrate $\mathrm{H}_{d}$. We wish that no site has more than one negative bond adjacent to it, because otherwise at least one plaquette will be overfrustrated. But there are two sites adjacent to one bond, so we need at least $2^{d-2} d$ sites. The ratio of needed sites (in order to fully frustrate $\mathrm{H}_{d}$ without overfrustrated plaquettes) to the number of available sites is

$$
R=\frac{d}{4}
$$

This proves that the overblocking effect takes place necessarily for simple cubic lattices above dimension 4 . For $d \leqslant 4$, it is easily checked by explicit construction that the overblocking does not take place so that the Ising ground state energy is given by formula (1). For $d=2$, we need only one negative bond to fully frustrate the square (Fig. 1); there are only two sites adjacent to one negative bond, so that the ratio $R=\frac{1}{2}$.

For $d=3$, we need at least three negative bonds (Fig. 2); there are six sites adjacent to one negative bond, so that the ratio $R=\frac{3}{4}$. For $d=4$, we need at least eight negative bonds (Fig. 3); there are sixteen sites adjacent to one negative bond, so that the ratio $R=1$. For $d>4$, the problem is more difficult as we have no simple way of knowing the minimal number of negative bonds, or the number of overfrustrated plaquettes (plaquettes with three negative bonds). The situation is more complicated because now a site may be adjacent to 0,1 or more negative bonds. We will construct later the minimal configuration of $\mathrm{H}_{5}, \mathrm{H}_{6}$ and $\mathrm{H}_{7}$, but before doing so we are going to find a lower bound for the ground state energy of $\mathrm{H}_{d}$.

Let us introduce for each configuration of a fully frustrated hypercube $\mathrm{H}_{d}$ the function $N(h)$. If $h$ is the difference between the number of positive and negative bonds adjacent to a given site in $\mathrm{H}_{d}, h$ is the inter- 


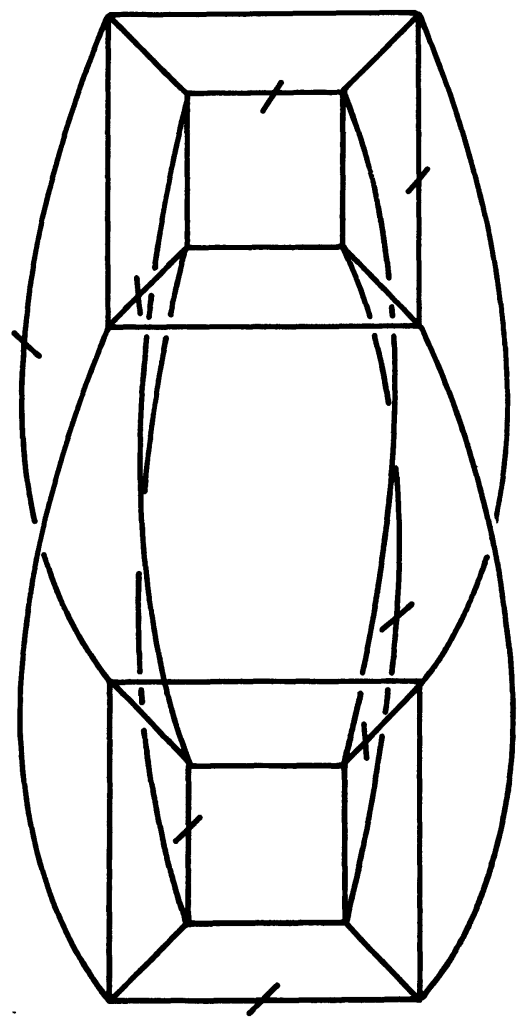

Fig. 3. - Fully frustrated $\mathrm{H}_{4}$ with eight negative bonds.

nal field created on this site by its neighbours. By definition, $N(h)$ is the number of sites in $\mathrm{H}_{d}$ in the field $h$.

It is obvious that for any site of $\mathrm{H}_{d}$, the only possible values of $h$ are $d, d-2, d-4, \ldots,-d$ and, as we are looking for the ground state, we cannot have negative values for $h$. This means that each spin is aligned with its own internal field.

Knowing this function $N(h)$, one can express the Ising ground state energy per unit $J$ and per spin in the lattice :

$$
E_{d}=-\frac{\sum_{h} h N(h)}{\sum_{h} N(h)}=-\bar{h} .
$$

(Note that this energy is twice the energy per spin of an isolated $\mathrm{H}_{d}$.)

Using the fact that all the plaquettes are frustrated, one can show that $\overline{h^{2}}=d$. If $h_{i}$ is the field created on the site $i$ by its neighbours, one has :

so

$$
h_{i}=\sum_{j} J_{i j} S_{j}
$$

$$
h_{i}^{2}=\sum_{j} J_{i j}^{2}+\sum_{j, k} J_{i j} J_{i k} S_{j} S_{k}
$$

When one sums over all the sites, the second term on the right side of this equation disappears as a consequence of the frustration of all the plaquettes, and one finds :

$$
\overline{h^{2}}=d=\frac{\sum h^{2} N(h)}{\sum N(h)} .
$$

This relation is true for any configuration of the spins and, in particular, gives a first lower bound for the ground state energy :

$$
E_{d}=-\bar{h} \geqslant-\left(\overline{h^{2}}\right)^{1 / 2}=-\sqrt{d} .
$$

(Note that this bound remains true for spins with any number of components.)

In the case of Ising spins, as the field $h_{i}$ takes only integer values (even values when $d$ is even, odd values when $d$ is odd), it is possible to find a better bound by looking for the distribution $N(h)$ verifying (4) which gives the lowest energy (2).

The problem we have to solve now is to find among all the distributions $N(h)$ verifying :

$$
\sum N(h)=2^{d}, \quad \sum h^{2} N(h)=d 2^{d},
$$

the distribution which minimizes

$$
E_{d}=-\frac{\sum h N(h)}{\sum N(h)},
$$

with the constraint that $N(h)$ takes integer values. Moreover, $h$ is positive or zero and takes only even (or odd) values when $d$ is even (or odd). As a matter of fact, the relation $\overline{h^{2}}=d$ is still true if the average is taken over only one half of the spins (We can divide the sites of $\mathrm{H}_{d}$ into two sets : set I and set II ; if a site belongs to set I, all its neighbours belong to set II) : spins of set I or spins of set II.

If $n(h)$ is the number of sites of one of these two subsets in the field $h$, our lower bound for the ground state energy is the lowest value of :

$$
E_{d}=-\frac{\sum h n(h)}{\sum n(h)}=-\bar{h},
$$

where the distribution $n(h)$ has to satisfy :

$$
\begin{aligned}
\sum h^{2} n(h) & =d 2^{d-1}, \\
\sum n(h) & =2^{d-1} .
\end{aligned}
$$

$\overline{h^{2}}$ is fixed, and we want to maximize $\bar{h}$. This means that the best distribution $n(h)$ will be as concentrated as possible. The best would be to have all the spins in the field $\sqrt{d}$ but when $\sqrt{d}$ is not integer, this is not possible and the distribution $n(h)$ is then concentrated on the 2,3 or 4 integers nearest to $\sqrt{d}$.

The best values of $E_{d}$ and the corresponding distributions $n(h)$ are given in table I for $d \leqslant 10$. 
We can see that, in general, when the distribution $n(h)$ is concentrated on the two integers nearest to $\sqrt{d}$, the solution is unique. When it is concentrated on 3 or 4 integers we find many solutions. This happens for $d=6$ and $d=8$.

Obviously, when $\sqrt{d}$ is an integer, the best distribution is $n(\sqrt{d})=2^{d-1}$.

Now the question is to determine whether this lower bound is reached by the true ground state energy. We have only been able to answer this question for $d \leqslant 7$, by giving an explicit construction of a fully frustrated $\mathrm{H}_{d}$.

For $d \leqslant 4$, one can verify that the minimal configurations (Figs. 1, 2 and 3) reach the energies given in table I. The distributions $n(h)$ are also the same.

Table I. - A lower bound for the ground state energy and the corresponding internal field distributions.

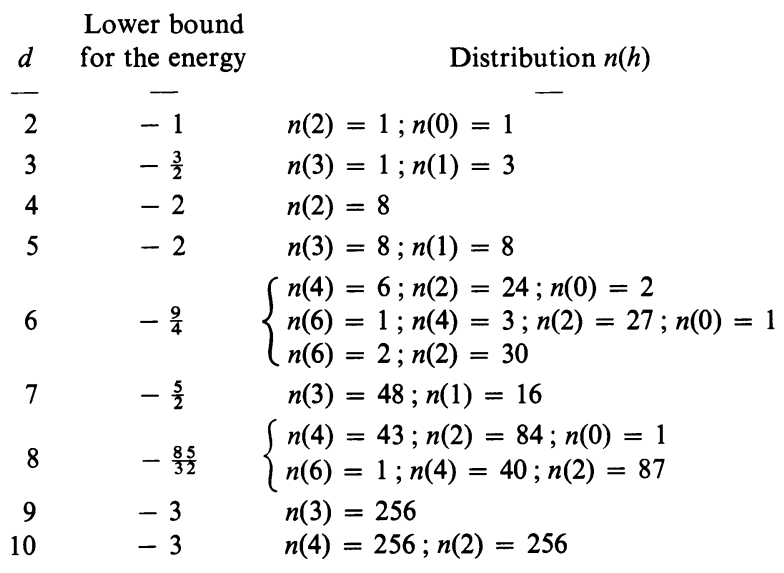

For $d>4$, we have attacked the problem by making drawings for $\mathrm{H}_{5} . \mathrm{H}_{5}$ contains 32 sites, 80 bonds, 80 plaquettes, 40 cubes and $10 \mathrm{H}_{4}$. We found experimentally a variety of fully frustrating configurations with 24 negative bonds and 8 triply frustrated plaquettes (the remaining 72 being simply frustrated). Among the sites, 16 were in a field $h=3$ and 16 in a field $h=1$. As the energy of this configuration reaches our lower bound (table I), we are sure that it is the ground state energy.

The four extra negative bonds, and the eight overfrustrated plaquettes, can be distributed more or less homogeneously in a minimal solution for $\mathrm{H}_{5}$. For commodity reasons, we shall consider a solution where the overfrustrated plaquettes are as closepacked as possible (Fig. 4). This solution can be seen as a square of supersites (cubes) linked by superbonds (bundles of eight bonds joining two supersite cubes). The four supersites are in minimal states (as far as their internal structure is concerned) with three negative bonds and nine positive bonds. Among the four superbonds, three are in minimal states, let us call them white, with two negative bonds, and six positive bonds; and one is in an excited state, let us call it black, with six negative bonds and two posi-

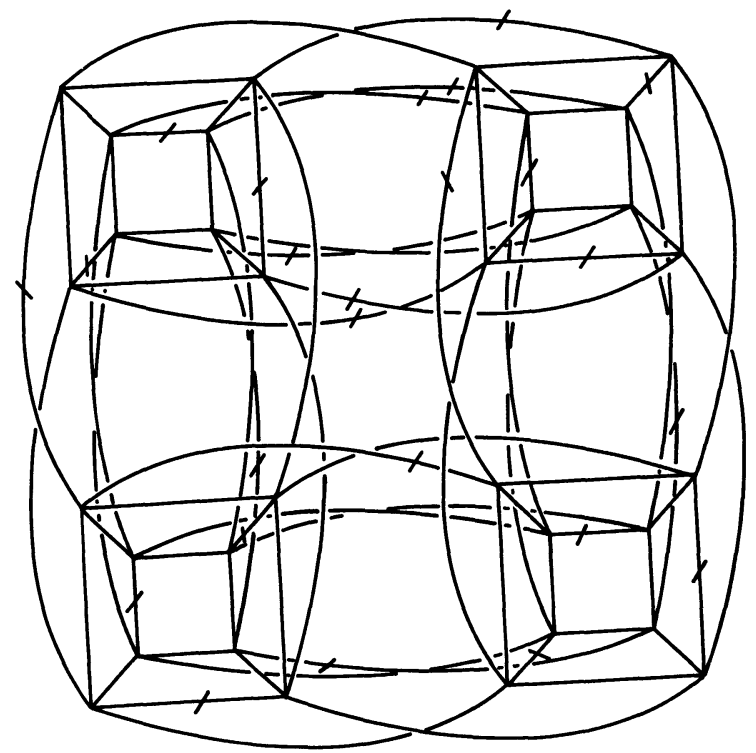

Fig. 4. - Fully frustrated $\mathrm{H}_{5}$ with twenty-four negative bonds. Eight plaquettes are overfrustrated (with three negative bonds).

tive bonds (Fig. 5). It is easily checked that such a square, with three white superbonds and one black superbonds : white go to black, and black to white). a minimal solution.

This solution is interesting because it has a scaling feature, which allows a renormalization procedure to be used to get to higher dimensions. Indeed $\mathrm{H}_{5}$, with its square of superbonds, is the analogue of $\mathrm{H}_{2}$, with its square of bonds, where white and black superbonds play the role of positive and negative bonds, respectively (Figs. 1, 5). Excluding for the moment sclutions with gray superbonds, it is easily seen that there is a frustration rule for a plaquette of superbonds (odd number of black superbonds) and a gauge invariance on supersites (a set of simultaneous gauge transformations on the eight sites of a supersite cube does not modify the frustration of the plaquettes and changes the character of the adjacent superbonds : white go to black, and black to white).

In the same way a fully frustrated configuration for $\mathrm{H}_{6}$ can be built from a minimal solution for the cube $\mathrm{H}_{3}$, where the eight sites become eight minimal

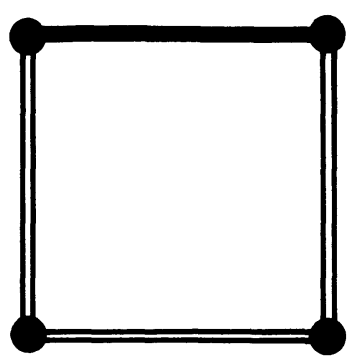

Fig. 5. - Same fully frustrated $\mathrm{H}_{5}$ as on figure 4, represented as a square of supersites (cubes) linked by superbonds. Three superbonds are white and one superbond is black. Compare with figure 1 


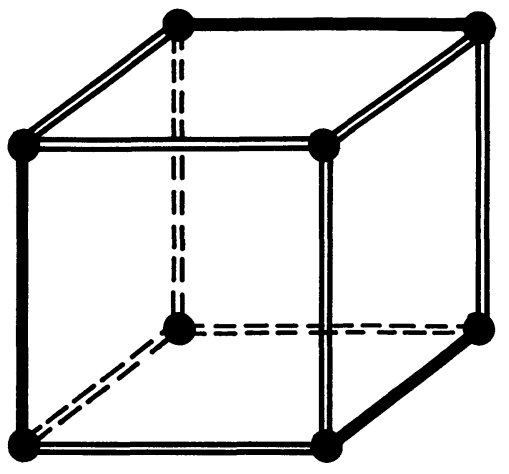

Fig. 6. - Fully frustrated $\mathbf{H}_{6}$, represented as a cube of supersites, with three black superbonds. Compare with figure 2.

supersites and the three negative bonds become three black superbonds (Fig. 6). This configuration for $\mathrm{H}_{6}$ contains 60 negative bonds (out of a total of 192) and 30 overfrustrated plaquettes (out of a total of 240). Clearly, this construction can be pursued in higher dimensions : for $d \geqslant 5$, a configuration for $\mathrm{H}_{d}$ can thus be built from a configuration for $\mathrm{H}_{d-3}$.

From this iterative construction, a recurrence relation for the energy of these configurations can be derived. From the formula

$$
E_{d}=-d+\frac{n_{d}}{2^{d-2}},
$$

where $n_{d}$ is the number of negative bonds in a minimal $\mathrm{H}_{d}$, and from the recurrence relation for bond numbers

$$
n_{d}=2^{d-3} \cdot d+4 \cdot n_{d-3}
$$

one gets the recurrence relation for the energies of these configurations

$$
E_{d}=\frac{E_{d-3}}{2}-\frac{3}{2}
$$

One can verify, using (7), that when $d \leqslant 7$, the energies of the so-constructed configurations are equal to our lower bound (table I), and so there is no doubt that our lower bound gives the true ground state energy.

As it was possible to deduce a recurrence relation for the energies of the configurations obtained by our iterative construction (7), one can likewise find for these configurations a recurrence law for the distributions $N_{d}(h)$ :

$$
N_{d+3}(h)=6 N_{d}(h-1)+2 N_{d}(3-h) .
$$

The distributions we find for $d \leqslant 7$ from this relation are compatible with the distributions given in table I. In the case of $d=6$, the configuration obtained by this construction corresponds to the first solution in table I $(n(4)=6 ; n(2)=24 ; n(0)=2)$. We do not know if the other solutions given in table I for $d=6$ are realizable.
For $d>8$, the energies given by (7) become strictly higher than our lower bound and when $d=9$ and $d>11$, it is possible to prove that the configurations constructed by our iterative method are no longer the ground states of the hypercube. Indeed, using (8), one finds that for $d=9, N(-1)=24$; this means that 24 spins are in a field -1 and so this configuration cannot be the ground state.

So for $d \geqslant 8$, we do not know what the ground state energy is, and for $d=9$ we are sure that this energy is lower than the energy given by (7). We present the conjecture that our lower bound always gives the ground state energy, but we have no rigorous proof of this because we do not know how to construct a fully frustrated $\mathrm{H}_{d}$ having this energy. For the moment, we can only prove a weaker result concerning the behaviour of the ground state energy when $d$ is large : $E_{d}$ is proportional to $\sqrt{d}$. Using the results of appendix $I$, it is possible to find an upper bound for the ground state energy. Thus, when $d$ is large, we have :

$$
-\sqrt{d} \leqslant E_{d} \leqslant-\sqrt{2 / \pi} \sqrt{d} .
$$

Let us remark that, as already mentioned, our iterative construction certainly fails for $d \geqslant 9$, but it was still useful in that it gives such a simple picture for the ground state, up to $d=7$ (Fig. 7).

One can imagine that a more powerful method would give the ground states in higher dimension. In the case of fcc lattices, a similar iterative construction gives the ground state energy in any dimension (see Appendix II).

In order to prepare the discussion and the comparison with other lattices, we shall reexpress some of these results in terms of the coordination number.

As mentioned in the previous section, from one minimal fully frustrating configuration for $\mathrm{H}_{d}$, we build a minimal fully frustrating configuration for the $d$-dimensional simple cubic lattice by reflexions with respect to the hyperplanes limiting $\mathrm{H}_{d}$. We then construct a periodic lattice where the unit cell contains $2^{d}$ hypercubes $\mathrm{H}_{d}$. The Ising ground state energy of the fully frustrated simple cubic lattice (per spin, per $J$ unit) can then be written as

$$
E_{d}=-\frac{\bar{h}}{2}
$$

where the internal field $h$ of a lattice site is the difference between the numbers of unfrustrated and frustrated bonds adjacent to the site and $\bar{h}$ is the average field taken over the fully frustrated lattice.

For $d \leqslant 4$, the average ground state internal field is $\bar{h}=d$, and thus $\bar{h}$ is proportional to the coordination number $z$ ( $=2 d$ for sc lattices). Note that, whereas for $d=2$ and $d=4$, homogeneous field states are realizable (same value for the field on each lattice site), in the case $d=3$, the ground states are necessarily mixed field states. 
For $d>4$, one finds from the asymptotic form of the ground state energy that it behaves (when $d$ is large) like the square root of the coordination number $z$.

At this stage, it is interesting to make a comparison with the results obtained for other lattices.

\section{Comparison with other fully frustrated lattices. -} Most prominent are the results for fcc lattices [3]. The diamond lattice in dimension three will be briefly discussed too.

In a $d$-dimensional fcc lattice, the coordination number is $z=2 d(d-1)$ and the number of bonds in a plaquette is $l=3$, so that the formula for the Ising ground state energy, in the absence of the overblocking effect, is

$$
E_{d}=-\frac{d(d-1)}{3}
$$

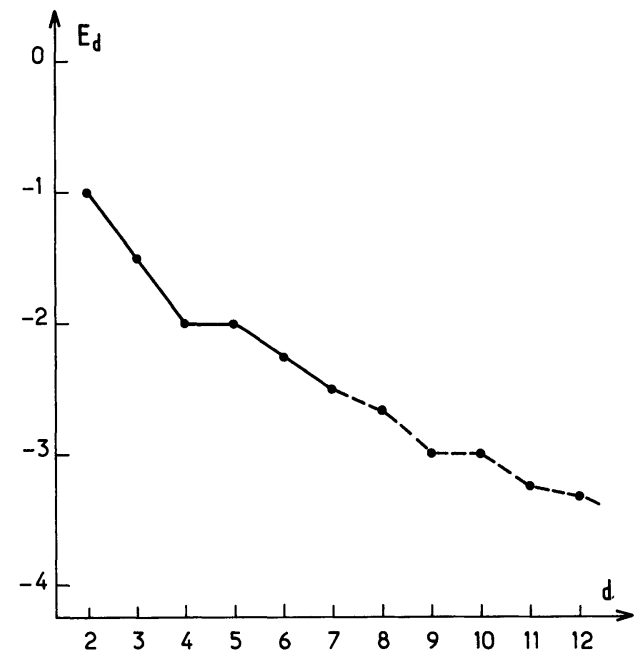

Fig. 7. - Plot of the Ising ground state energy $E_{d}$ for a fully frustrated simple cubic lattice as a function of the dimensionality for $d \leqslant 7$. The dotted line represents our lower bound for $d \geqslant 8$ which we conjecture to be the true ground state energy.

This formula has been shown by Alexander-Pincus to hold true for $d \leqslant 4$. In terms of the average internal field $\bar{h}$ one obtains

$$
\bar{h}=\frac{2 d(d-1)}{3} \text {. }
$$

Here also for $d \leqslant 4, \bar{h}$ is proportional to $z$.

For $d>4$, Alexander-Pincus have found

$$
\begin{array}{ll}
E_{d}=-d, & \text { so that } \bar{h}=2 d, \text { for } d \text { even } \\
E_{d}=-(d-1), & \text { so that } \bar{h}=2(d-1),
\end{array}
$$

for $d$ odd

so

$$
\bar{h} \sim \sqrt{2 z} \text { when } d \rightarrow \infty .
$$

The common features are the onset of the over- blocking effect above dimension four and the behaviour of the average internal field.

Because the relation between the coordination number $z$ and the dimension $d$ is qualitatively different in the two lattices, the common asymptotic behaviour for the average internal field as a function of dimension yields necessarily qualitatively different behaviour for other quantities. Thus, the Ising ground state energy (per spin), which decreases linearly with dimension (above dimension 4) for fcc lattices, decreases like $-\sqrt{d}$ for sc lattices.

With this in mind, we go back to the study of the fully frustrated diamond lattice (in dimension three) made by J. Villain [2]. In this study, he conjectured a ground state Ising energy

$$
E=-1 \text {, }
$$

in the units defined above (which are half of Villain's units). However, the energy formula, in the absence of the overblocking effect, predicts

$$
E=-\frac{4}{3},
$$

and indeed, a careful inspection of Villain's figure 3 shows that his solution contains overfrustrated plaquettes. Therefore, his conjecture appeared to contradict our statement about the universality of the onset of overblocking above dimension 4. But actually, the conjecture is unverified and the contradiction vanishes. In order to show this, we give an explicit construction of a minimal fully frustrating configuration of bonds for the diamond lattice. Consider the family of parallel dense (001) planes (horizontal planes on Villain's figure 3 ); each bond links two sites on neighbour planes $(n, n+1)$. Now consider all bonds between planes 0 and 1 , say, and make half of them negative and half of them positive in an alternating way; then, take all bonds between planes +1 and +2 and between planes +2 and +3 as positive; now again, between planes +3 and +4 , make half of the bonds negative and half positive in an alternating way, and iterate the construction. It is easily checked that this configuration fully frustrates the lattice without overfrustration, and the corresponding Ising ground state energy is $E=-\frac{4}{3}$, as expected.

From our constructions of three-dimensional fully frustrated lattices, consequences can be drawn for the ground state degeneracies, which are now examined.

4. Ground state degeneracies of the fully frustrated diamond, fec and sc lattices. - For the diamond lattice, in the construction given above for the ground state, there is a binary choice every third plane. This implies a ground state entropy, which is of onedimensional character,

$$
S_{0} \sim N^{1 / 3}
$$

where $N$ is the number of sites. 
That is the behaviour found also for the threedimensional fcc lattice. In both cases, an Ising ground state can be constructed by a random one-dimensional stacking of two-dimensional sheets.

However, for the three-dimensional sc lattice, it appears that the behaviour is different. Starting from one ground state configuration, it appears that there is a two-dimensional array of parallel columns (one column out of four belongs to this array) which can be flipped independently, all sites belonging to one linear column being flipped simultaneously. This implies a ground state entropy, which is at least of two-dimensional character,

$$
S_{0} \sim N^{2 / 3} .
$$

In summary, there is a high ground state degeneracy for these three lattices, albeit no residual entropy in the thermodynamic limit $\left(S_{0} / N \rightarrow 0\right.$, as $\left.N \rightarrow \infty\right)$. But the degeneracy is qualitatively higher for the sc lattice than for the fcc and diamond lattices in dimension 3 .

It is important and physically instructive to know whether these three-dimensional fully frustrated models have a finite transition temperature. In the absence of any precise result, we can give the following plausibility discussion.

As discussed by various authors, it appears plausible that the fcc lattice has a finite $T_{\mathrm{c}}$, because a ground state is made by stacking two-dimensional unfrustrated square lattices which have by themselves a finite $T$. The same plausibility argument applies for the diamond lattice, but note that the stacked sheets are now made of rather loosely bound square lattices. For the sc lattice, the existence of a $T_{\mathrm{c}}$ becomes a very moot question, due to the higher degeneracy. Further work is needed.

In higher dimension, Alexander and Pincus found for fcc lattices a ground state entropy proportional to $N^{1 / d}$.

In the case of sc lattices, the situation is certainly less regular : we could only draw definite conclusions for $d=4$ and $d=6$.

For $d=4$, one can prove that there are oniy 16 ground states : if one chooses the frustrated bonds for only one $\mathrm{H}_{3}$ in this $4 d$ lattice, all the bonds of the lattice are fixed.

For $d=6$, we constructed a ground state for the lattice with a finite proportion of spins in a zero field (see table I). This means that for $d=6$, the ground state entropy is macroscopic.

5. Perspectives. - It is of general interest to investigate the behaviour of phase transitions in arbitrary dimension, because one wishes to determine the upper and lower characteristic dimensionalities around which a renormalization group expansion is possible. Moreover, here, it is of interest to know whether there is, at least in some dimensionality range, a continuity between the fully frustrated phase and the spin glass phase [3]. In order to answer this question, it is natural to determine first what are the universal features of the fully frustrated phase. Our study of sc lattices, coming after the study of fcc lattices by $\mathrm{S}$. Alexander and P. Pincus, has shown the generality of the overblocking effect and of the behaviour of the average internal field above dimension four. This behaviour is remarkably similar to that of spin glasses : the square root dependence in $z$ evokes irresistibly the square root law for random fluctuations in the central limit theorem, and many phenomenological arguments which have been devised for spin glasses [5] should be tried and worked out on the overblocked fully frustrated phases. Further work on the transition temperatures, and on the nature of the low temperature phases, appears desirable. Finally, the bond percolation problem on such fully frustrated lattices appears as a natural way to introduce the disorder and to get to spin glass models [4].

Appendix I. - In this appendix, we construct a family of configurations for $\mathrm{H}_{d}$, whose energies per spin behave like $-\sqrt{\frac{2}{\pi} d}$. when $d$ is large.

Let us come back to the first construction of $\mathrm{H}_{d}$ given in the body of the paper; we take two opposite fully frustrated $\mathrm{H}_{d-1}$ linked by $2^{d-1}$ positive bonds (if a bond is positive in one of the two $\mathrm{H}_{d-1}$, the corresponding bond is negative in the other $\mathrm{H}_{d-1}$ ).

As we start from $\mathrm{H}_{2}$, this construction preserves the symmetry between the two subsets of sites : set I and set II. This means that the distribution $n(h)$ is the same for these two sets in any dimension. One can deduce from this construction the recurrence relation :

$$
n_{d+1}(h)=n_{d}(h-1)+n_{d}(1-h) .
$$

If we start from $d=2$ with $n(0)=1$ and $n(2)=1$, one can verify that in dimension $d, n(h)$ is non vanishing only for $h=d, d-2, d-4, \ldots, 2-d$ and is given by :

$$
n_{d}(d-2 p)=C_{d-1}^{p} .
$$

The corresponding energy of these configurations is :

$$
E_{d}=-\frac{\sum h n(h)}{\sum n(h)}=-1 \text {. }
$$

When $d \geqslant 3$, the configurations constructed by this method have some sites in a negative field.

If we make a gauge transformation for all the spins of set I which are in a negative field, the corresponding energies of these new configurations are :

$$
E_{d}=-\frac{\sum|h| n(h)}{\sum n(h)}
$$


From (A. 1) we can find the recurrence relation for this energy :

$$
\begin{array}{ll}
E_{d+1}=E_{d}-2^{-d-1} C_{d}^{d / 2}, & \text { if } d \text { is even }, \\
E_{d+1}=E_{d}, & \text { if } d \text { is odd } .
\end{array}
$$

Using the asymptotic form of $C_{d}^{d / 2}$ :

$$
C_{d}^{d / 2} \sim 2^{d} \sqrt{\frac{2}{\pi d}},
$$

we find

$$
E_{d} \sim-\sqrt{\frac{2}{\pi} d} .
$$

Note that this is only an upper bound for the ground state energy : for instance when $d=4$, the true energy is -1 and this construction gives only $-\frac{3}{4}$.

Appendix II. - In this appendix, we recover part of the results of Alexander and Pincus [3] by the scaling method used in the body of this paper for the simple cubic lattices. The structure under study is an Ising system on an antiferromagnetic face centred cubic (fcc) lattice.

As in the simple cubic case, it is only necessary to find the ground state of the unit cell of the lattice, which is then extended by successive reflexions with respect to the hyperplanes limiting the unit cell. The unit cell of the fcc lattice in dimension $d$ is found as follows : one selects half of the vertices (say the thick ones) on $\mathrm{H}_{d}$, in such a way that two thick vertices are never connected by a bond of $\mathrm{H}_{d}$. Each plaquette of $\mathrm{H}_{d}$

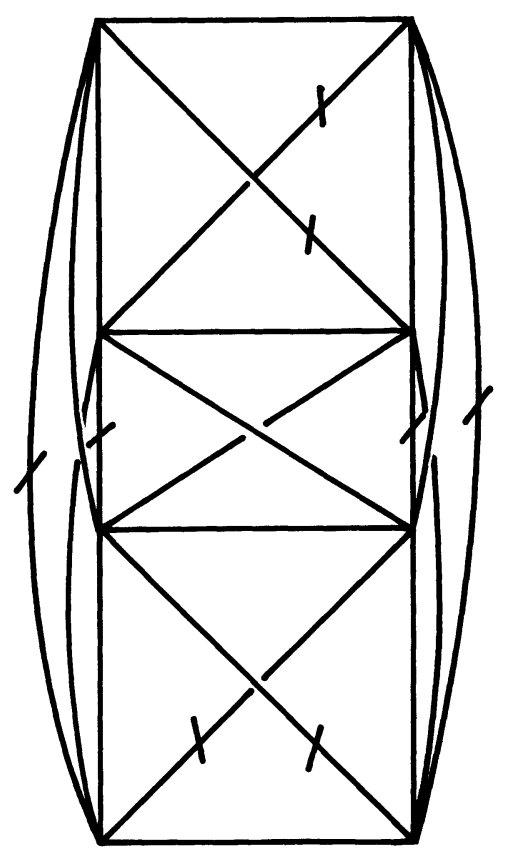

Fig. 8. - Fully frustrated unit cell of the face centred cubic lattice in dimension 4.

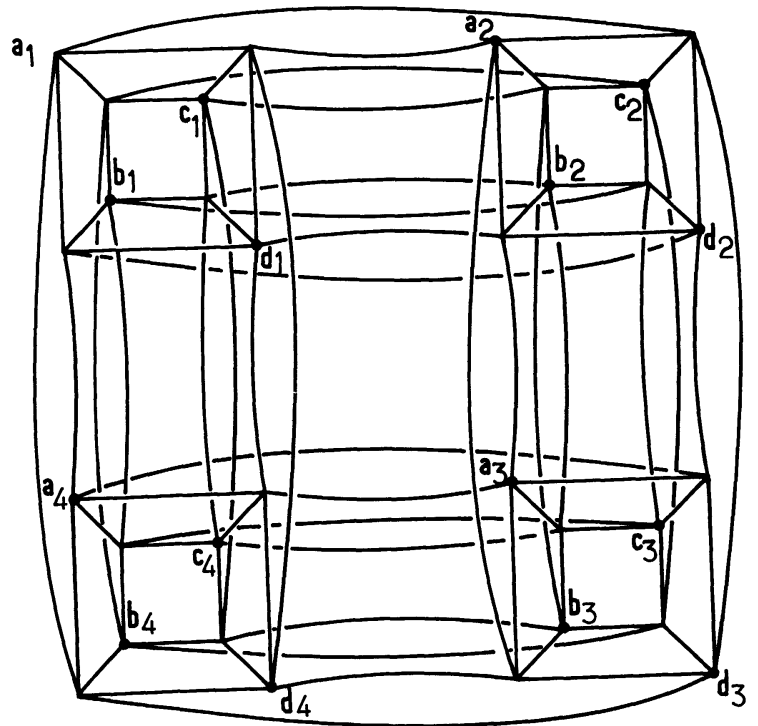

Fig. 9. - Unit cell of the face centred cubic lattice in dimension 5 . The thick vertices belong to a $\mathrm{H}_{5}$ and are labelled $a_{1}, b_{1}, c_{1}, d_{1}$; $a_{2}, b_{2}, c_{2}, d_{2}, \ldots$

has two thick vertices diametrically opposed, and the sought-for unit cell is obtained by drawing a link between any pair of thick vertices on the same plaquette of $\mathrm{H}_{d}$.

One gets in this way a tetrahedron from $\mathrm{H}_{3}$; in figure 8 , we have drawn the result of this construction for $d=4$, with the indication of a possible configuration of minimum energy; in figure 9 , we have drawn the thick vertices on $\mathrm{H}_{5}$, they form four groups of four vertices numbered $a_{1} b_{1} c_{1} d_{1} ; a_{2} b_{2} c_{2} d_{2}, \ldots$

The next task is to divide this set of 16 thick vertices (on $\mathrm{H}_{5}$ ) into four sets of four vertices. Each of them will constitute a supervertex and the bundle of bonds between two such supervertices will be a superbond. In this way, we shall recover a tetrahedral structure in the fcc cell in $d=5$, and thus find by renormalization procedure the ground state in any dimension.

An elementary inspection shows that it is not possible to select as supervertices the groups $a_{1} b_{1} c_{1} d_{1}$, $\begin{array}{lllll}a_{2} & b_{2} & c_{2} & d_{2}, \ldots & \text { because the bundles connecting }\end{array}$ $\left(a_{1} b_{1} c_{1} d_{1}\right)$ to $\left(a_{2} b_{2} c_{2} d_{2}\right)$ and $\left(a_{1} b_{1} c_{1} d_{1}\right)$ to $\left(a_{3} b_{3} c_{3} d_{3}\right)$ are different. A possible choice for the supervertices is $\left(a_{1} b_{2} d_{3} c_{4}\right),\left(b_{1} a_{2} c_{3} d_{4}\right),\left(c_{1} d_{2} b_{3} a_{4}\right)$ and $\left(d_{1} c_{2} a_{3} b_{4}\right)$.

In each supervertex, there are two internal bonds $\left[a_{1} d_{3}\right.$ and $b_{2} c_{4}$ in $\left.\left(a_{1} b_{2} d_{3} c_{4}\right)\right]$ and each vertex is connected by a bond to 3 vertices of any other supervertex (for instance $a_{1}$ is connected to $a_{2}, d_{4}, b_{1}$; $c_{1}, d_{2}, a_{4}$; and to $d_{1}, c_{2}, d_{4}$. Remember that in the fcc lattice $a_{1}$ is connected with any thick vertex with which it shares a common plaquette in $\mathrm{H}_{5}$ ). Each supervertex of four vertices is connected with any other supervertex through a bundle (or superbond) of 12 bonds; and from each vertex start 1 (internal 
bond in the supervertex) $+3 \times 3$ (external bonds) $=$ 10 bonds, which is indeed the number of plaquettes through a given vertex in $\mathrm{H}_{5}$.

Now a symmetric fully frustrated state of the fcc cell is constructed as follows : each internal bond is frustrated, a superbond is (non) frustrated or black (white), when each of its 12 bonds are (non) frustrated. As the superbonds and supervertices have the structure of a tetrahedron, one keeps two black superbonds and four white superbonds with the same arrangement as the frustrated and non frustrated bonds in a fully frustrated tetrahedron.

With this choice, 6 non frustrated bonds (those of the 2 white superbonds) and 4 frustrated bonds ( $=3$ of a black superbond +1 internal bond) start from each vertex. Thus, $n_{+} / n_{-}=\frac{3}{2}$ and the internal field of any vertex is $h=n_{+}-n_{-}=z / 5$ $\left[z=n_{+}+n_{-}\right.$is the coordination number $=$ $2 d(d-1)=40]$ and the energy per spin is $E=-4$, in agreement with the findings of Alexander and Pincus.

A straightforward extension of this construction allows one to recover the even-odd alternation in $\mathrm{E}_{d}$, since the ground state at $d=5$ is found by identification of a supertetrahedron, which is the unit cell in $d=3$.

\section{References}

[1] Toulouse, G., Commun. Phys. 2 (1977) 115.

[2] Villain, J., J. Phys. C 10 (1977) 1717.

[3] Alexander, S. and Pincus, P., preprint.

[4] DI Si LE, L., J. Phys. C 10 (1977) L-353.

[5] Anderson, P. W., Pond, C. M., Phys. Rev. Lett. 40 (1978) 903. 